\title{
Chirality switching of an antiferromagnetic spiral wall and its effect on magnetic anisotropy
}

\author{
Q. Li, ${ }^{1}$ M. Yang, ${ }^{1}$ A. T. N'Diaye, ${ }^{2}$ C. Klewe, ${ }^{2}$ P. Shafer, ${ }^{2}$ N. Gao, ${ }^{1}$ T. Y. Wang, ${ }^{1}$ E. Arenholz,${ }^{3}$ Xixiang Zhang, ${ }^{4}$ \\ C. Hwang, ${ }^{5} \mathrm{~J} . \mathrm{Li},{ }^{6,}{ }^{,}$and Z. Q. Qiu ${ }^{1, \dagger}$ \\ ${ }^{1}$ Department of Physics, University of California at Berkeley, Berkeley, California 94720, USA \\ ${ }^{2}$ Advanced Light Source, Lawrence Berkeley National Laboratory, Berkeley, California 94720, USA \\ ${ }^{3}$ Cornell High Energy Synchrotron Source, Cornell University, Ithaca, New York 14853, USA \\ ${ }^{4}$ Physical Science and Engineering Division (PSE), King Abdullah University of Science and Technology (KAUST), \\ Thuwal 23955-6900, Saudi Arabia \\ ${ }^{5}$ Korea Research Institute of Standards and Science, Yuseong, Daejeon 305-340, Korea \\ ${ }^{6}$ International Center for Quantum Materials, School of Physics, Peking University, Beijing 100871, China
}

(Received 13 September 2019; published 26 November 2019)

\begin{abstract}
An antiferromagnetic $\mathrm{NiO}$ spiral wall in $\mathrm{Fe} / \mathrm{NiO} / \mathrm{Co}_{0.5} \mathrm{Ni}_{0.5} \mathrm{O} /$ vicinal $\mathrm{Ag}(001)$ was created by rotating $\mathrm{Fe}$ magnetization and investigated using x-ray magnetic linear dichroism (XMLD). Different from the Mauri's $180^{\circ}$ spiral wall, we find that the $\mathrm{NiO}$ spiral wall always switches its chirality at $\sim 90^{\circ}$ rotation of the $\mathrm{Fe}$ magnetization, and unwinds the spiral wall back to a single domain with a further rotation of the Fe magnetization from $90^{\circ}$ to $180^{\circ}$. The effect of this chirality switching on the magnetic anisotropy was studied using rotational magneto-optic Kerr effect (ROTMOKE) on $\mathrm{Py} / \mathrm{NiO} / \mathrm{Co}_{0.5} \mathrm{Ni}_{0.5} \mathrm{O} /$ vicinal $\mathrm{Ag}(001)$. We find that the original Mauri's model has to be corrected by an energy folding due to the chirality switching, which consequently converts the exchange bias from the Mauri's $180^{\circ}$ spiral wall into a uniaxial anisotropy and a negative fourfold anisotropy.
\end{abstract}

DOI: 10.1103/PhysRevMaterials.3.114415

A ferromagnetic (FM)/antiferromagnetic (AFM) system is one of the most interesting magnetic systems because of the induced exchange bias and magnetic anisotropy in the FM layer [1-3]. The key issue of this subject is how the AFM spins respond to the FM magnetization reversal and subsequently affect the FM layer properties? Despite the great progress in terms of material expansion [4] and measurement scale [5], there exist many ambiguous issues mostly due to the lack of a direct measurement of the AFM spins so that most studies have to assume certain AFM spin configurations [6-10]. This difficulty is overcome partially by the development of $\mathrm{x}$-ray magnetic linear dichroism (XMLD) [11] which could probe AFM spins in certain AFM single crystalline films. For example, it has been believed for a long time that exchange bias comes only from FM/AFM interfacial coupling [12] until it was shown that the exchange bias actually depends on AFM bulk spins [13-15] and could even be dominated by AFM defects [16]. This mystery was resolved by XMLD measurement which shows that exchange bias is associated only with a small amount of pinned uncompensated AFM spins [17] which could have a wide distribution from the FM/AFM interface to the bulk AFM layer [18]. In contrast, the majority compensated AFM spins, depending on their rotatable or frozen nature [19], are responsible for different types of the magnetic anisotropies in the FM layer [20,21]. Regarding the key question of how the AFM spins respond to the FM magnetization reversal, the most mysterious issue concerns the so-called Mauri's model in which FM/AFM interfacial

\footnotetext{
*Corresponding author: jiali83@pku.edu.cn

†Corresponding author: qiu@berkeley.edu
}

coupling is presumed to twist the AFM spins into a $180^{\circ}$ spiral wall during the FM magnetization reversal and subsequently creates an exchange bias [22]. In theory, Mauri's model has encountered the difficulty of AFM interfacial spin frustrations $[23,24]$, and should lead to a zero exchange bias in the limit of perfect spin compensation [25]. In experiment, a recent XMLD measurement challenges the Mauri's $180^{\circ}$ spiral wall structure by showing that the AFM spiral wall winds up only to $\sim 90^{\circ}$, then switches its chirality, and unwinds the wall from $90^{\circ}$ to $180^{\circ}$ rotation of the FM magnetization [26]. Noticing that Mauri's model has been applied to a FM/AFM system over three decades, the absence of the Mauri's $180^{\circ}$ wall raises an urgent question about whether the Mauri's model needs a correction by the chirality switching of the AFM spiral wall in order to appropriately describe the FM overlayer? To answer this question, we carried out an experimental study on $\mathrm{FM} / \mathrm{NiO} / \mathrm{Co}_{0.5} \mathrm{Ni}_{0.5} \mathrm{O} /$ vicinal $\mathrm{Ag}(001)$. By rotating the $\mathrm{FM}$ magnetization, we revealed the $\mathrm{NiO}$ spiral wall structure by XMLD measurement and studied the corresponding magnetic anisotropies of the FM layer by rotation magneto-optic Kerr effect (ROTMOKE).

A $10 \mathrm{~mm} \times 10 \mathrm{~mm}$ vicinal $\left(6^{\circ}\right.$ angle) $\mathrm{Ag}(001)$ with steps parallel to [110] axis [Fig. 1(a)] was cleaned in an ultrahigh vacuum system by cycles of $\mathrm{Ar}$ ion sputtering at $2 \mathrm{keV}$ and annealing at $500{ }^{\circ} \mathrm{C}$. An $8-\mathrm{nm} \mathrm{Co}_{0.5} \mathrm{Ni}_{0.5} \mathrm{O}$ film was grown by coevaporating $\mathrm{Co}$ and $\mathrm{Ni}$ at $200{ }^{\circ} \mathrm{C}$ substrate temperature and at an oxygen pressure of $2.0 \times 10^{-6}$ Torr, followed by an annealing at $450{ }^{\circ} \mathrm{C}$ for half an hour in an oxygen environment. Then a $\mathrm{NiO}$ wedge $(0-18 \mathrm{~nm})$ was deposited by moving the substrate behind a shutter [27] at the oxygen atmosphere of $2.0 \times 10^{-6}$ Torr at room temperature. A 1.5$\mathrm{nm} \mathrm{Fe}$ film was deposited on one half of the sample for 
(a)

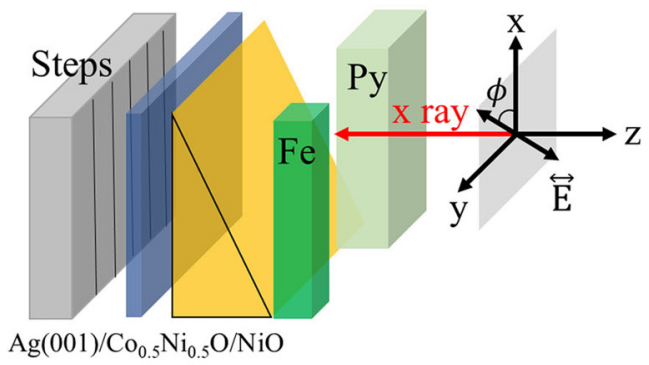

(b)

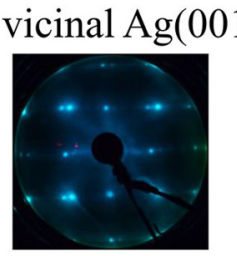

$\mathrm{NiO}$

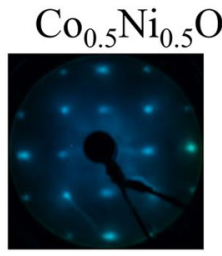

$\mathrm{Fe}$
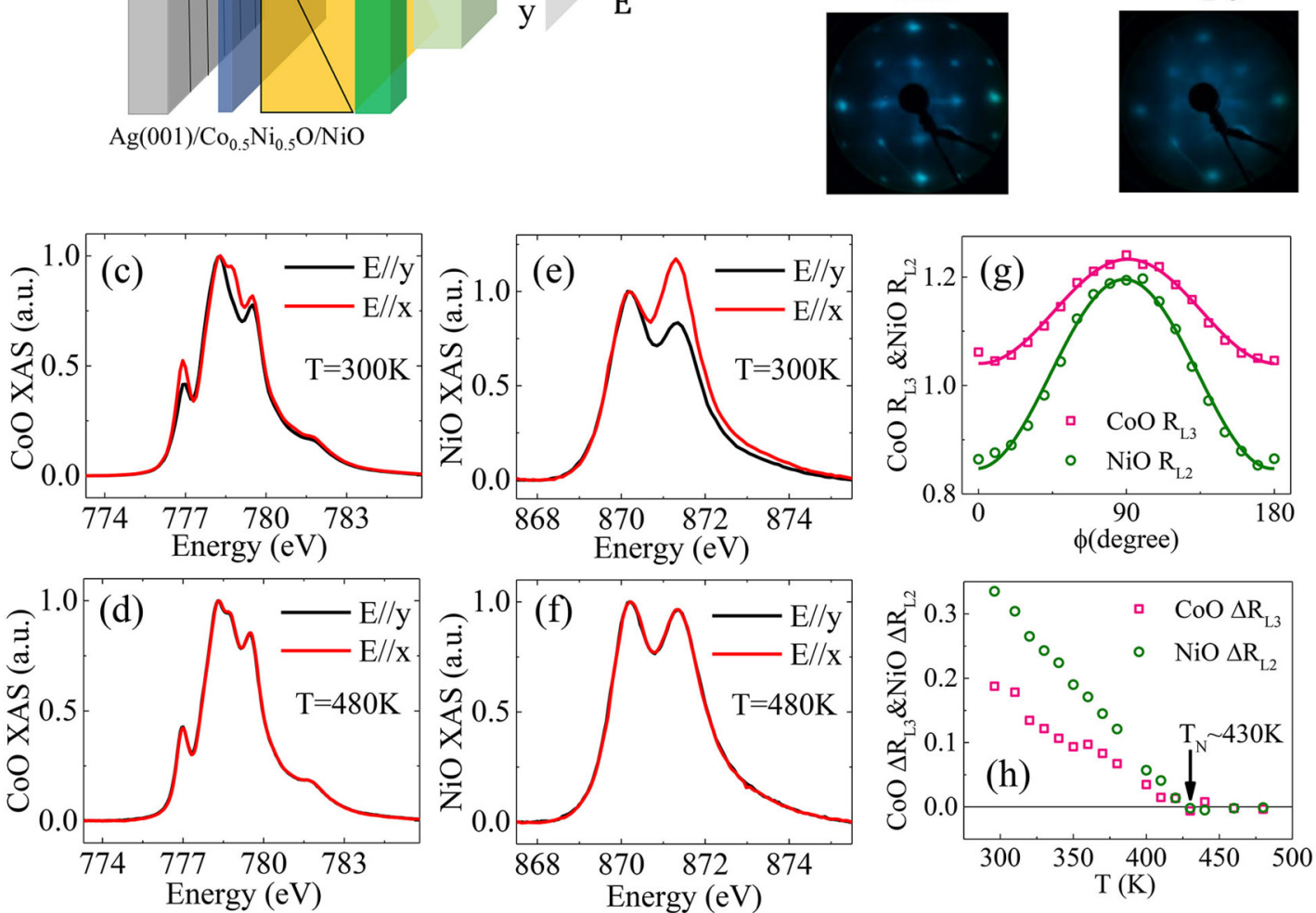

FIG. 1. (a) Schematic drawing of the sample and XMLD measurement. (b) LEED patterns of $\mathrm{Fe}(1.5 \mathrm{~nm}) / \mathrm{NiO}(10 \mathrm{~nm}) / \mathrm{Co} 0.5 \mathrm{Ni}{ }_{0.5} \mathrm{O}(8 \mathrm{~nm}) /$ vicinal $\mathrm{Ag}(001)$. XAS from $\mathrm{Fe}(1.5 \mathrm{~nm}) / \mathrm{Co}_{0.5} \mathrm{Ni}_{0.5} \mathrm{O}(8 \mathrm{~nm}) /$ vicinal $\mathrm{Ag}(001)$ with the x-ray polarization parallel $(\stackrel{\leftrightarrow}{E} / / x)$ and perpendicular to steps $(\stackrel{\leftrightarrow}{E} / / y)$ taken from (c) and (d) the Co $L_{3}$ edge and (e) and (f) the Ni $L_{2}$ edge. (g) Both the Co $L_{3}$ ratio and the Ni $L_{2}$ ratio exhibit cos ${ }^{2} \phi$ dependence on the $\mathrm{x}$-ray polarization angle $(\phi)$ at $T=300 \mathrm{~K}$, showing that both Co and $\mathrm{Ni}$ spins in $\mathrm{Co}_{0.5} \mathrm{Ni}_{0.5} \mathrm{O}$ are along the $y$ axis. (h) Both Co $L_{3}$ ratio and $\mathrm{Ni} L_{2}$ ratio differences vanish at $430 \mathrm{~K}$, showing $T_{\mathrm{N}}=430 \mathrm{~K}$ for the $8 \mathrm{~nm} \mathrm{Co}_{0.5} \mathrm{Ni}_{0.5} \mathrm{O}$ film.

XMLD measurement and a 25-nm Py film was deposited on the other half of the sample for ROTMOKE measurement. Both $\mathrm{Fe}$ and Py were grown at room temperature. The Py layer was evaporated from a thermal crucible with mixed target in the composition of $\mathrm{Fe}_{20} \mathrm{Ni}_{80}$. A low energy electron diffraction (LEED) pattern [Fig. 1(b)] shows the formation of single crystalline $\mathrm{Co}_{0.5} \mathrm{Ni}_{0.5} \mathrm{O}, \mathrm{NiO}$, and $\mathrm{Fe}$ films with $\mathrm{Fe}[100] / / \mathrm{NiO}[110] / / \mathrm{Co}_{0.5} \mathrm{Ni}_{0.5} \mathrm{O}[110] / / \mathrm{Ag}[110]$, and the formation of polycrystalline Py film. The sample was capped with a 2-nm Ag layer [28], and was measured using XMLD in total electron yield mode at beam line 4.0.2 and beamline 6.3.1 of the Advanced Light Source (ALS). ROTMOKE was measured using a rotation MOKE setup [29].

We first took Co and Ni X-ray absorption spectra (XAS) in $\mathrm{Fe} / \mathrm{Co}_{0.5} \mathrm{Ni}_{0.5} \mathrm{O} /$ vicinal $\mathrm{Ag}(001)$. It is known that a vicinal surface aligns the AFM spin axis for $\mathrm{CoO}(001)$ and $\mathrm{NiO}(001)$ films $[27,30]$. The different Néel temperatures $\left(T_{\mathrm{N}}\right)$ of $\mathrm{CoO}$ $\left(T_{\mathrm{N}} \sim 290\right)$ and $\mathrm{NiO}\left(T_{\mathrm{N}} \sim 520 \mathrm{~K}\right)$ are expected to lead to an AFM $\mathrm{Co}_{0.5} \mathrm{Ni}_{0.5} \mathrm{O}$ film on vicinal $\mathrm{Ag}(001)$ with $T_{\mathrm{N}}>300 \mathrm{~K}$
[31-33] and with an in-plane spin axis perpendicular to the atomic steps [27]. By taking XAS [19-21,26-28] from the Co $L_{3}$ edge [Figs. $1(\mathrm{c})$ and $\left.1(\mathrm{~d})\right]$ and the $\mathrm{Ni} L_{2}$ edge [Figs. 1(e) and 1(f)] at normal incidence of the $\mathrm{x}$ rays, the XMLD effect manifests as the different XAS for X-ray polarization $(\stackrel{\leftrightarrow}{E})$ parallel to the $x$ and $y$ axis, respectively. Then the presence of the XMLD at $300 \mathrm{~K}$ [Figs. 1(c) and 1(e)] and the absence of XMLD at $480 \mathrm{~K}$ [Figs. 1(d) and 1(f)] indicate that the 8-nm $\mathrm{Co}_{0.5} \mathrm{Ni}_{0.5} \mathrm{O}$ film is AFM ordered at room temperature. The Co $R_{L 3}$ ratio (intensity at $E \sim 778.3 \mathrm{eV}$ divided by intensity at $E \sim 778.8 \mathrm{eV}$ ) and $\mathrm{Ni} R_{L 2}$ ratio (intensity at $E \sim 870.2 \mathrm{eV}$ divided by intensity at $E \sim 871.3 \mathrm{eV}$ ) [Fig. 1(g)] exhibit the expected $\cos ^{2} \phi$ dependence on the $\mathrm{X}$-ray polarization angle $(\phi)$. Both the $\mathrm{Co} R_{L 3}$ and $\mathrm{Ni} R_{L 2}$ at $300 \mathrm{~K}$ exhibit maximum at $\phi=90^{\circ}$, showing that both Co and Ni AFM spins are perpendicular to the atomic steps (parallel to the $y$ axis). Both the Co $L_{3}$ ratio difference $\left[\Delta R_{L 3} \equiv R_{L 3}(\stackrel{\leftrightarrow}{E} \| y)-\right.$ $R_{L 3}(\stackrel{\leftrightarrow}{E} \| x)$ and Ni $L_{2}$ ratio difference $\left[\Delta R_{L 2} \equiv R_{L 2}(\stackrel{\leftrightarrow}{E} \| y)-\right.$ 

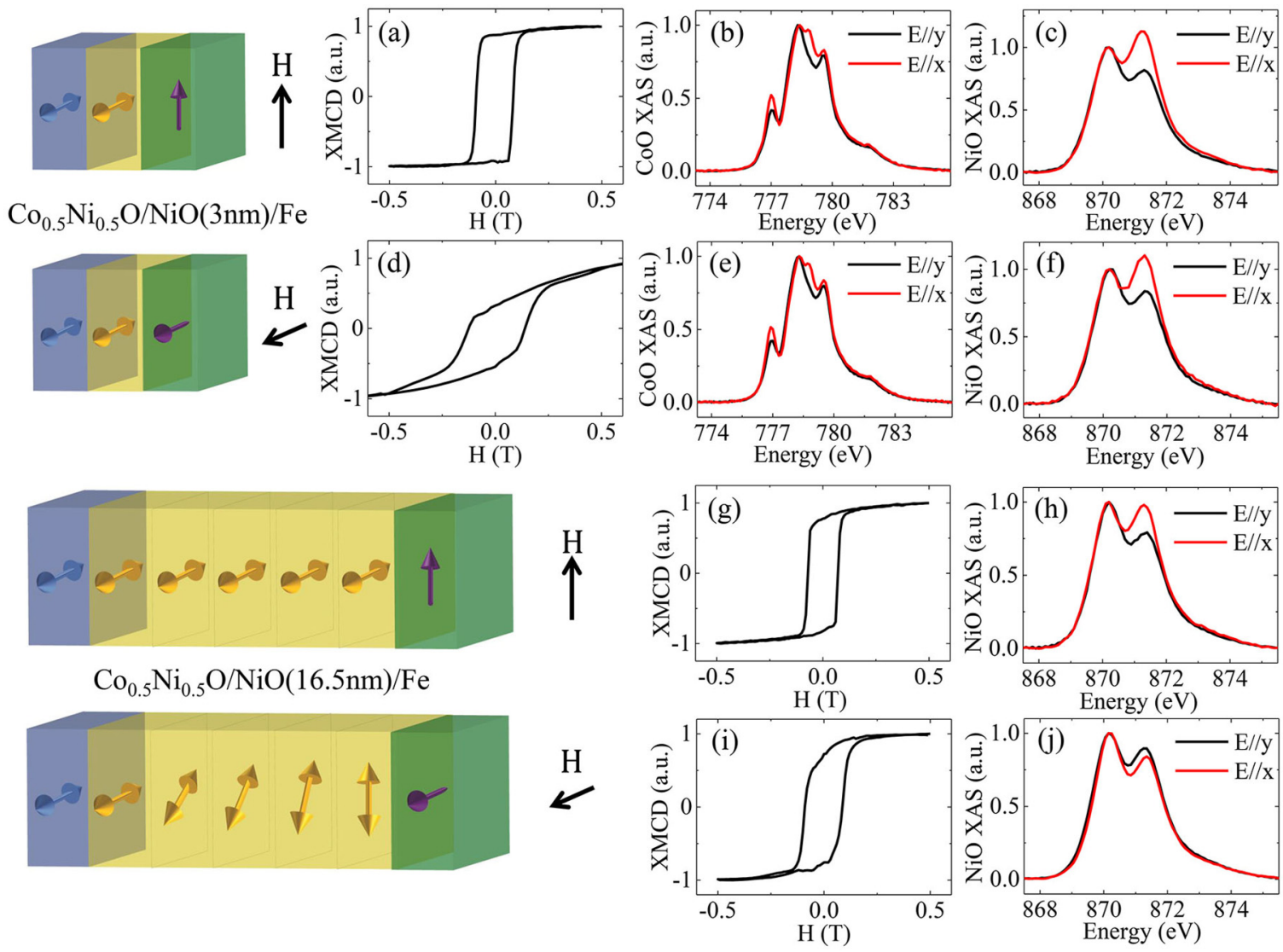

FIG. 2. Result from $\mathrm{Fe}(1.5 \mathrm{~nm}) / \mathrm{NiO}\left(d_{\mathrm{NiO}}\right) / \mathrm{Co}_{0.5} \mathrm{Ni}_{0.5} \mathrm{O}(8 \mathrm{~nm}) /$ vicinal $\mathrm{Ag}(001)$. Fe hysteresis loops for (a) and (g) $\vec{H} / / x$ and $(\mathrm{d})$ and (i) $\vec{H} / / y$ show that the $\mathrm{Fe}$ and $\mathrm{NiO}$ spins are coupled perpendicularly. By aligning the Fe magnetization along the $x$ and $y$ axis, (b) and (e) Co XAS and (c) and (f) Ni XAS show that both the $\mathrm{Co}_{0.5} \mathrm{Ni}_{0.5} \mathrm{O}$ and the $\mathrm{NiO}$ AFM spins in $d_{\mathrm{NiO}}=3 \mathrm{~nm}$ samples are frozen to the $y$ axis. (h) and (j) Ni XAS from the $d_{\mathrm{NiO}}=16.5 \mathrm{~nm}$ sample show that the $\mathrm{NiO}$ AFM spins are twisted into a spiral wall after the rotation of Fe magnetization from the $x$ axis to the $y$ axis.

$\left.R_{L 2}(\stackrel{\leftrightarrow}{E} \| x)\right]$ decrease with temperature and vanish at $430 \mathrm{~K}$, showing that the $\mathrm{Co}$ and $\mathrm{Ni}$ spins in $\mathrm{Co}_{0.5} \mathrm{Ni}_{0.5} \mathrm{O}$ are AFM ordered together with a Néel temperature of $T_{\mathrm{N}}=430 \mathrm{~K}$ [Fig. 1(h)].

We then studied the $\mathrm{NiO}$ spiral wall in $\mathrm{Fe}(1.5 \mathrm{~nm}) /$ $\mathrm{NiO}\left(d_{\mathrm{NiO}}\right) / \mathrm{Co}_{0.5} \mathrm{Ni}_{0.5} \mathrm{O}(8 \mathrm{~nm}) /$ vicinal $\mathrm{Ag}(001)$ by applying a 4000 Oe magnetic field $(\vec{H})$ to align the Fe magnetization to the $x$ and $y$ axis, respectively. In the thin limit of $\mathrm{NiO}$ thickness at $d_{\mathrm{NiO}}=3 \mathrm{~nm}$, we observe the same XAS spectra at Co $L_{3}$ edge [Figs. 2(b) and 2(e)] for $\vec{H} / / x$ and $\vec{H} / / y$, showing that the $\mathrm{Co}_{0.5} \mathrm{Ni}_{0.5} \mathrm{O}$ spins are frozen to the $y$ axis and do not rotate with the Fe magnetization. Similarly, the same XAS spectra at Ni $L_{2}$ edge for $\vec{H} / / x$ and $\vec{H} / / y$ [Figs. 2(c) and 2(f)] show that the $3 \mathrm{~nm} \mathrm{NiO}$ spins are also frozen, i.e., the $\mathrm{NiO} / \mathrm{Co}_{0.5} \mathrm{Ni}_{0.5} \mathrm{O}$ interfacial coupling is much stronger than the $\mathrm{Fe} / \mathrm{NiO}$ interfacial coupling to pin the 3-nm $\mathrm{NiO}$ spins parallel to the $\mathrm{Co}_{0.5} \mathrm{Ni}_{0.5} \mathrm{O}$ spins. In addition, the $\mathrm{Fe}$ easy-axis hysteresis loop for $\vec{H} / / x$ [Fig. 2(a)] and hard-axis loop for $\vec{H} / / y$ [Fig. 2(d)] show that the Fe magnetization is coupled perpendicularly to the $\mathrm{NiO}$ spins, in agreement to the spin-flop coupling at the $\mathrm{Fe} / \mathrm{NiO}$ interface $[19,28,34]$. In the thick limit of $d_{\mathrm{NiO}}=16.5 \mathrm{~nm}$, the NiO XAS [Fig. 2(h)] shows the same character as that in the $d_{\mathrm{NiO}}=3 \mathrm{~nm}$ sample [Fig. 2(e)] for $\vec{H} / / x$, but reverses its sign [Fig. 2(j)] after the
Fe magnetization is rotated from its easy $x$ axis to its hard $y$ axis. Taking into account the $5.5 \mathrm{~nm}$ probing depth of an electron yield in $\mathrm{NiO}$ [27] and of the $\mathrm{NiO}$ spin pinning at the $\mathrm{NiO} / \mathrm{Co}_{0.5} \mathrm{Ni}_{0.5} \mathrm{O}$ interface, the results of Figs. 2(h) and $2(\mathrm{j})$ suggest that the $\mathrm{Fe} / \mathrm{NiO}$ perpendicular interfacial coupling has twisted the $16.5-\mathrm{nm} \mathrm{NiO}$ AFM spins into a spiral wall at $\vec{H} / / y$ with the $\mathrm{NiO}$ spins roughly parallel to the $x$ axis at the $\mathrm{Fe} / \mathrm{NiO}$ interface and pinned to the $y$ axis at the $\mathrm{NiO} / \mathrm{Co}_{0.5} \mathrm{Ni}_{0.5} \mathrm{O}$ interface.

Next, we measured the NiO XMLD in $\mathrm{Fe}(1.5 \mathrm{~nm}) /$ $\mathrm{NiO}$ (wedge) $/ \mathrm{Co}_{0.5} \mathrm{Ni}_{0.5} \mathrm{O}(8 \mathrm{~nm}) /$ vicinal $\mathrm{Ag}(001)$ where the $\mathrm{NiO}$ wedge ensures the same $\mathrm{Fe} / \mathrm{NiO}$ interface so that the XMLD result should reveal systematically the dependence of the $\mathrm{NiO}$ spiral wall on $d_{\mathrm{NiO}}$. We rotate the $\mathrm{Fe}$ magnetization (rotation angle $\varphi_{\mathrm{FM}}$ ) with a 4000 Oe rotating magnetic field and measured the $\mathrm{NiO} L_{2}$ ratio difference $\left[\Delta R_{L 2} \equiv\right.$ $R_{L 2}(\stackrel{\leftrightarrow}{E} \| y)-R_{L 2}(\stackrel{\leftrightarrow}{E} \| x)$ ] as a function of $\varphi_{\mathrm{FM}}$ [Fig. 3(a)]. For 3-nm NiO film, the $\Delta R_{L 2}$ value remains unchanged with increasing $\varphi_{\mathrm{FM}}$ [Fig. 3(b)], confirming the frozen NiO AFM spins for $d_{\mathrm{NiO}}<3 \mathrm{~nm}$. For thicker NiO films, the $\Delta R_{L 2}\left(\varphi_{\mathrm{FM}}\right)$ value decreases as $\varphi_{\mathrm{FM}}$ increases from $0^{\circ}$ to $90^{\circ}$, showing a winding of the $\mathrm{NiO}$ spins into a spiral wall. As $\varphi_{\mathrm{FM}}$ keeps increasing from $90^{\circ}$ to $180^{\circ}$, the $\Delta R_{L 2}\left(\varphi_{\mathrm{FM}}\right)$ value gradually increases to resume its original value of $\Delta R_{L 2}\left(0^{\circ}\right)$ 

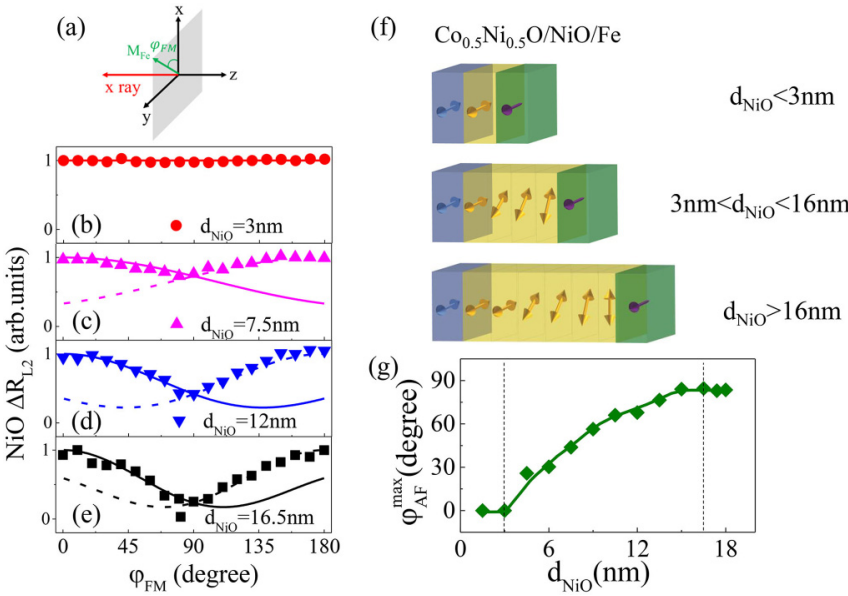

FIG. 3. (a) Schematic drawing of the XMLD measurement. (b)(e) $\mathrm{NiO} \Delta R_{L 2}$ as a function of the Fe magnetization rotation angle $\left(\varphi_{\mathrm{FM}}\right)$. The solid and dashed lines are calculated XMLD for righthanded and left-handed spiral walls, respectively. (f) Schematic drawing of the $\mathrm{NiO}$ AFM spiral walls in three $d_{\mathrm{NiO}}$ regions. (g) $\mathrm{NiO}$ spiral angle as a function of $d_{\mathrm{NiO}}$, where $\varphi_{\mathrm{AF}}^{\max }$ is the $\mathrm{NiO}$ spin rotation angle at $\mathrm{Fe} / \mathrm{NiO}$ interface at $\varphi_{\mathrm{FM}}=90^{\circ}$.

[Figs. 3(c)-3(e)]. The symmetric behavior of $\Delta R_{L 2}\left(\varphi_{\mathrm{FM}}\right)$ with respect to $\varphi_{\mathrm{FM}}=90^{\circ}$ indicates that the $\mathrm{NiO}$ spiral wall has switched its chirality from right handed to left handed at $\varphi_{\mathrm{FM}}=90^{\circ}$ and unwinds back to single domain as the $\mathrm{Fe}$ magnetization rotates from $90^{\circ}$ to $180^{\circ}$ [26].

To obtain the $\mathrm{NiO}$ spiral angle $\varphi_{\mathrm{AF}}^{\max }$ (the $\mathrm{NiO}$ spin rotation angle at the $\mathrm{Fe} / \mathrm{NiO}$ interface at $\varphi_{\mathrm{FM}}=90^{\circ}$ ), we consider the $\mathrm{NiO}$ XMLD signal as the averaged result from the $\mathrm{NiO}$ spiral spins at different depth $\Delta R_{L 2}=\frac{1}{d_{\mathrm{NiO}}} \int_{0}^{d_{\mathrm{NiO}}} \Delta R_{L 2}(z) e^{-d / \lambda} d z$, where $d$ is the distance from the ${ }^{\mathrm{Ne}} \mathrm{Fe} / \mathrm{NiO}$ interface and $\lambda=5.5 \mathrm{~nm}$ is the electron escape depth $[26,27]$. The value of $\mathrm{NiO} \Delta R_{L 2}$ for different $\mathrm{NiO}$ thicknesses were rescaled here to follow a general fitting formula. Assuming a linear dependence of the $\mathrm{NiO}$ spin rotation angle on $d$, $\varphi(d)=\varphi_{\mathrm{AF}}^{\max }\left(d_{\mathrm{NiO}}-d\right) / d_{\mathrm{NiO}}$ and the NiO XMLD signal of $\Delta R_{L 2}(d)=A \cos ^{2} \varphi(d)+B$, we calculated the averaged $\Delta R_{L 2}$ for right-handed and left-handed spiral walls and fitted the experimental data with $\varphi_{\mathrm{AF}}^{\max }$ as the only fitting parameter. The result [solid and dashed lines in Figs. 3(b)-3(e)] agrees very well with the experimental data. The retrieved $\varphi_{\mathrm{AF}}^{\max }[$ Fig. $3(\mathrm{~g})]$ shows that the NiO AFM spins are frozen for $d_{\mathrm{NiO}}<3 \mathrm{~nm}$, wind up to a spiral wall for $3<d_{\mathrm{NiO}}<16 \mathrm{~nm}$, and saturate to the final $\varphi_{\mathrm{AF}}^{\max } \sim 84^{\circ}$ spiral wall for $d_{\mathrm{NiO}}>16 \mathrm{~nm}$. Regardless of the $\varphi_{\mathrm{AF}}^{\max }$ value, we always observe a chirality switching at $\varphi_{\mathrm{FM}}=90^{\circ}$, showing that Mauri's $180^{\circ}$ spiral wall never forms in $\mathrm{NiO}$ film in the whole thickness range.

Next, we studied the FM overlayer magnetic anisotropy. In order to reveal the induced magnetic anisotropy rather than the enhancement of an existing anisotropy, we studied Py soft magnetic film as opposed to Fe film. Since the $\mathrm{Py} / \mathrm{NiO}$ coupling has comparable coupling strength with the $\mathrm{Fe} / \mathrm{NiO}[35,36]$, we expect that the $\mathrm{NiO}$ spiral wall in $\mathrm{Py} / \mathrm{NiO} / \mathrm{Co}_{0.5} \mathrm{Ni}_{0.5} \mathrm{O} /$ vicinal $\mathrm{Ag}(001)$ behaves the same as in $\mathrm{Fe} / \mathrm{NiO} / \mathrm{Co}_{0.5} \mathrm{Ni}_{0.5} \mathrm{O} /$ vicinal $\mathrm{Ag}(001)$. We first confirmed that the 25-nm Py layer on vicinal $\operatorname{Ag}(001)$ has a negli- (a)

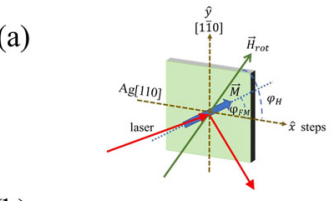

(b)

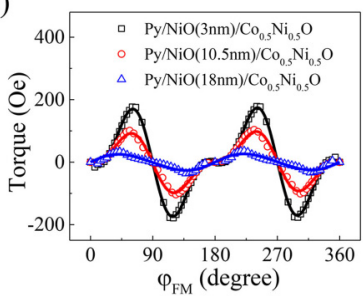

(c)

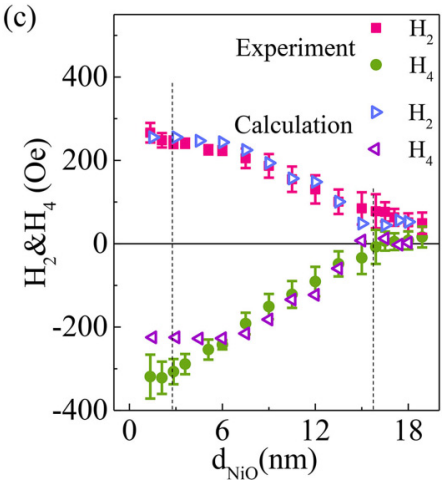

FIG. 4. (a) Experiment geometry for ROTMOKE measurement. (b) Magnetic torque as a function of Py magnetization angle $\left(\varphi_{\mathrm{FM}}\right)$ from $\mathrm{Py}(25 \mathrm{~nm}) / \mathrm{NiO}\left(d_{\mathrm{NiO}}\right) / \mathrm{Co}_{0.5} \mathrm{Ni}_{0.5} \mathrm{O}(8 \mathrm{~nm}) /$ vicinal $\mathrm{Ag}(001)$. Solid lines are fitting results using Eq. (2). (c) Py uniaxial and fourfold anisotropies as a function of $d_{\mathrm{NiO}}$.

gible magnetic anisotropy so that any Py anisotropies in Py/NiO/ $/ \mathrm{Co}_{0.5} \mathrm{Ni}_{0.5} \mathrm{O} /$ vicinal $\mathrm{Ag}(001)$ should come entirely from the $\mathrm{Py} / \mathrm{NiO}$ interaction. ROTMOKE was applied to the film with a 700-Oe in-plane rotating magnetic field. The Py magnetic energy density is described by

$$
\begin{aligned}
E= & -M H \cos \left(\varphi_{H}-\varphi_{\mathrm{FM}}\right)-M H_{1} \cos \varphi_{\mathrm{FM}}+\frac{1}{2} M H_{2} \sin ^{2} \varphi_{\mathrm{FM}} \\
& +\frac{1}{2} M H_{4} \sin ^{2} \varphi_{\mathrm{FM}} \cos ^{2} \varphi_{\mathrm{FM}} .
\end{aligned}
$$

Here $\varphi_{H}$ and $\varphi_{\mathrm{FM}}$ are the in-plane angles of the magnetic field $(H)$ and the Py magnetization $(M)$ with respect to the $x$ axis, respectively. $H_{1}, H_{2}$, and $H_{4}$ are the exchange bias, uniaxial anisotropy field, and fourfold anisotropy field, respectively. Under this definition, $H_{2}>0$ corresponds to the Py uniaxial easy axis being the $x$ axis, and $H_{4}>0$ corresponds to the Py fourfold easy axes being the $\pm x$ and $\pm y$ axes. Minimizing the energy with respect to $\varphi_{\mathrm{FM}}$ leads to

$$
\begin{aligned}
l\left(\varphi_{\mathrm{FM}}\right) \equiv & H \sin \left(\varphi_{H}-\varphi_{\mathrm{FM}}\right)=H_{1} \sin \varphi_{\mathrm{FM}}+\frac{1}{2} H_{2} \sin \left(2 \varphi_{\mathrm{FM}}\right) \\
& +\frac{1}{4} H_{4} \sin \left(4 \varphi_{\mathrm{FM}}\right) .
\end{aligned}
$$

Here $l\left(\varphi_{\mathrm{FM}}\right)$ is the magnetic torque which is determined by experimental values of $\varphi_{H}$ and $\varphi_{\mathrm{FM}}$. Figure 4(b) shows representative torque curves as a function of $\varphi_{\mathrm{FM}}$ from $\mathrm{Py}(25$ $\mathrm{nm}) / \mathrm{NiO}\left(d_{\mathrm{NiO}}\right) / \mathrm{Co}_{0.5} \mathrm{Ni}_{0.5} \mathrm{O}(8 \mathrm{~nm}) / \mathrm{vicinal} \mathrm{Ag}(001)$ at $d_{\mathrm{NiO}}=$ $3,10.5$, and $18 \mathrm{~nm}$. By fitting the torque curves using Eq. (2), we obtained the anisotropy fields.

First, we find that the exchange bias field is negligibly small $\left(H_{1}<5 \mathrm{Oe}\right)$ for all $d_{\mathrm{NiO}}$, showing that the NiO chiralityswitched spiral wall (Fig. 3) does not lead to an obvious exchange bias. Next, we find that the Py film always consists of a positive uniaxial anisotropy $\left(H_{2}>0\right)$ and a negative fourfold anisotropy $\left(H_{4}<0\right)$ with their magnitudes being constants for $d_{\mathrm{NiO}}<3 \mathrm{~nm}$, decreasing with $d_{\mathrm{NiO}}$ for $3<d_{\mathrm{NiO}}<$ $16 \mathrm{~nm}$, and becoming small constants for $d_{\mathrm{NiO}}>16 \mathrm{~nm}$ [Fig. 4(c)], corresponding exactly to the three regions of the $\mathrm{NiO}$ chirality-switched spiral wall [Fig. 3(g)]. It should be mentioned that the planar domain wall width in bulk $\mathrm{NiO}$ was reported to be $\sim 14 \mathrm{~nm}$ [37] which is very close to the $\sim 16 \mathrm{~nm}$ value in our system. 
It is easy to understand the uniaxial anisotropy. For frozen $\mathrm{NiO}$ spins parallel to the $y$ axis at $d_{\mathrm{NiO}}<3 \mathrm{~nm}$, the $\mathrm{Py} / \mathrm{NiO}$ perpendicular interfacial coupling favors the Py magnetization in the $\pm x$ axis, leading to a uniaxial anisotropy. For $3<$ $d_{\mathrm{NiO}}<16 \mathrm{~nm}$, a rotation of the Py magnetization away from its easy $x$ axis should twist the $\mathrm{NiO}$ spins into a spiral wall. Because of the chirality switching of the $\mathrm{NiO}$ spiral wall at $\varphi_{\mathrm{FM}}=90^{\circ}$, the $\mathrm{NiO}$ spiral wall energy reaches maximum at $\varphi_{\mathrm{FM}}=90^{\circ}$ and then decreases for $90^{\circ}<\varphi_{\mathrm{FM}}<180^{\circ}$ due to the unwinding of the spiral wall. Noticing that the Mauri's $180^{\circ}$ spiral wall winds up all the way to $\varphi_{\mathrm{FM}}=180^{\circ}$, the chirality switching of the AFM spiral wall at $\varphi_{\mathrm{FM}}=90^{\circ}$ should convert the exchange bias of the Mauri's $180^{\circ}$ wall to a uniaxial anisotropy. For $d_{\mathrm{NiO}}>16 \mathrm{~nm}$, only $\mathrm{NiO}$ spins within $16 \mathrm{~nm}$ from the $\mathrm{Py} / \mathrm{NiO}$ interface wind up into a spiral wall, resulting in a constant spiral wall energy. Comparing to the relatively simple picture of the uniaxial anisotropy, the Py fourfold anisotropy is a little bit puzzling. Unlike $\mathrm{Fe} / \mathrm{CoO}(001)[20,38]$ where the $\mathrm{Fe} / \mathrm{CoO}$ magnetic coupling modifies the existing Fe fourfold anisotropy, polycrystalline Py film does not contain a fourfold anisotropy so that the observed $\mathrm{H}_{4}$ must come entirely from the Py/NiO interaction. It is tempting to relate the Py fourfold anisotropy to the $\mathrm{NiO}(001)$ fourfold anisotropy. However, bulk $\mathrm{NiO}$ and $\mathrm{CoO}$ anisotropies favor their spins parallel to the [110] and [110] axes ( $x$ and $y$ axes in Fig. 4) [25,38], thus they cannot explain the negative Py fourfold anisotropy which favors the Py spins parallel to the $\mathrm{NiO}[100]$ and [010] axes $\left( \pm 45^{\circ}\right.$ to the $x$ axis).

To understand the role of the chirality switching of the $\mathrm{NiO}$ spiral wall in the Py anisotropy, we have to correct the Mauri's model to describe the Py magnetic energy

$$
\mathrm{E}\left(\varphi_{\mathrm{FM}}\right)=E_{W}\left(1-\cos \varphi_{\mathrm{AF}}\right)+J_{C} \sin ^{2}\left(\varphi_{\mathrm{FM}}-\varphi_{\mathrm{AF}}\right),
$$

where the first term describes the $\mathrm{NiO}$ spiral wall energy, the second term describes the $\mathrm{Py} / \mathrm{NiO}$ perpendicular coupling, and $\varphi_{\mathrm{AF}}$ is the $\mathrm{NiO}$ spiral wall opening angle. To best single out the effect of the chirality switching of the AFM spiral wall, Eq. (3) only made two corrections to the original Mauri's model [22]: (1) the linear FM/AFM interfacial coupling in the Mauri's model is replaced by the perpendicular FM/AFM coupling; and (2) the chirality switching of the $\mathrm{NiO}$ spiral wall at $\varphi_{\mathrm{FM}}=90^{\circ}$ folds the energy from $0^{\circ}<\varphi_{\mathrm{FM}}<90^{\circ}$ into $90^{\circ}<\varphi_{\mathrm{FM}}<180^{\circ}$, i.e., $\mathrm{E}\left(\varphi_{\mathrm{FM}}\right)=\mathrm{E}\left(180^{\circ}-\varphi_{\mathrm{FM}}\right)$ for $90^{\circ} \leqslant \varphi_{\mathrm{FM}} \leqslant 180^{\circ}$. As shown in the Supplemental Material [39], it is the perpendicular FM/AFM coupling that permits a chirality switching of the AFM spiral wall at $\varphi_{\mathrm{FM}}=90^{\circ}$, leading to the energy folding which eliminates the exchange bias from the spiral wall (then exchange bias in real experimental systems are related to uncompensated spins, pinning centers, and defects, etc. [25] rather than the AFM spiral wall). Finally, $E_{W}=\sqrt{A K}$ in the original Mauri's model ( $A$ and $K$ are the $\mathrm{NiO}$ exchange stiffness and the anisotropy constant, respectively) should be valid only for $d_{\mathrm{NiO}}>16 \mathrm{~nm}$. For $d_{\mathrm{NiO}}<16 \mathrm{~nm}, E_{W}$ should depend on $d_{\mathrm{NiO}}$ and be greater than $\sqrt{A K}$. The $E_{W}$ value for $d_{\mathrm{NiO}}<16 \mathrm{~nm}$ can be retrieved by experimental value of $\varphi_{\mathrm{AF}}^{\max }$ in Fig. 3(g). In fact, it is easy to derive $E_{W}=2 J_{C} \cos \varphi_{\mathrm{AF}}^{\max }$ (valid for $d_{\mathrm{NiO}}>3 \mathrm{~nm}$ ) by minimizing Eq. (3) with respect to $\varphi_{\mathrm{AF}}$ at $\varphi_{\mathrm{FM}}=90^{\circ}$.
Noticing that it is Eq. (3) that leads to the anisotropy terms in Eq. (1), it is easy to recognize that $\Delta E \equiv E\left(\varphi_{\mathrm{FM}}=90^{\circ}\right)-$ $E\left(\varphi_{\mathrm{FM}}=0^{\circ}\right)$ is nothing but the uniaxial anisotropy. Then from $\varphi_{\mathrm{AF}} \approx 0^{\circ}$ for $d_{\mathrm{NiO}}<3 \mathrm{~nm}$ and $E_{W}=2 J_{C} \cos \varphi_{\mathrm{AF}}^{\max }$ for $d_{\mathrm{NiO}}>3 \mathrm{~nm}$, we obtain $H_{2} \approx H_{2}^{0} \equiv \frac{2 \mathrm{~J}_{\mathrm{C}}}{M}$ for $d_{\mathrm{NiO}}<3 \mathrm{~nm}$ and $H_{2}=H_{2}^{0}\left(2 \cos \varphi_{\mathrm{AF}}^{\max }-\cos ^{2} \varphi_{\mathrm{AF}}^{\max }\right)$ for $d_{\mathrm{NiO}}>3 \mathrm{~nm}$. Taking $\varphi_{\mathrm{AF}}^{\max }$ from Fig. 3(g), we plot the thickness dependence of $H_{2}\left(d_{\mathrm{NiO}}\right)$ in Fig. 4(c). The result agrees very well with the experimental data.

To understand the fourfold anisotropy especially in thin $\mathrm{NiO}$ thickness limit, consider first the extreme case of a small spiral angle. Minimizing the energy of Eq. (3) with respect to $\varphi_{\mathrm{AF}}$, it is easy to show that the total energy in this limit is $\mathrm{E} \approx J_{C} \sin ^{2} \varphi_{\mathrm{FM}}-2 J_{C}^{2} / E_{W} \sin ^{2} \varphi_{\mathrm{FM}} \cos ^{2} \varphi_{\mathrm{FM}}$, i.e., a positive uniaxial anisotropy and a negative fourfold anisotropy, in agreement with the experimental observation. In fact, the negative fourfold anisotropy is a general result of any small angle AFM spiral wall opened by a perpendicular FM/AFM interfacial coupling, and the energy folding at $\varphi_{\mathrm{FM}}=90^{\circ}$ due to the chirality switching of the AFM spiral wall naturally converts the exchange bias from the Mauri's $180^{\circ}$ spiral wall into a uniaxial, a fourfold, and higher even order anisotropies in the FM film (Supplemental Material [39]). For greater value of $\varphi_{\mathrm{AF}}$ in the region of $3<d_{\mathrm{NiO}}<16 \mathrm{~nm}$, we performed numerical calculation to minimize the energy of Eq. (3) and obtained the fourfold anisotropy $H_{4}$ by doing the corresponding Fourie transformation (Supplemental Material [39]). The result agrees well with the experimental data [Fig. 4(c)].

In summary, we investigated the $\mathrm{NiO}$ spiral wall in $\mathrm{FM} / \mathrm{NiO} / \mathrm{Co}_{0.5} \mathrm{Ni}_{0.5} \mathrm{O} /$ vicinal $\mathrm{Ag}(001)$ system. We find that $\mathrm{NiO}$ AFM spins are almost frozen for $d_{\mathrm{NiO}}<3 \mathrm{~nm}$, gradually form a spiral wall for $3<d_{\mathrm{NiO}}<16 \mathrm{~nm}$, and complete the spiral wall formation for $d_{\mathrm{NiO}}>16 \mathrm{~nm}$. Different from Mauri's $180^{\circ}$ wall, we find that the $\mathrm{NiO}$ spiral wall switches its chirality at $\varphi_{\mathrm{FM}}=90^{\circ}$ and consequently lead to a uniaxial anisotropy and a negative fourfold anisotropy. The original Mauri's model has to be corrected by taking into account the chirality switching of the AFM spiral wall in order to explain the experimental observations.

This work is supported by the U.S. Department of Energy, Office of Science, Office of Basic Energy Sciences, Materials Sciences and Engineering Division under Contract No. DEAC02-05CH11231 (van der Waals heterostructures program, KCWF16), National Science Foundation Grant No. DMR1504568, King Abdullah University of Science and Technology (KAUST), Office of Sponsored Research (OSR) and under the Award No. OSR-2016-CRG5-2977, Future Materials Discovery Program through the National Research Foundation of Korea (No. 2015M3D1A1070467), Science Research Center Program through the National Research Foundation of Korea (No. 2015R1A5A1009962), National Key Research and Development Program of China (No. 2016YFA0300804 and No. 2017YFA0303303). The operations of the Advanced Light Source at Lawrence Berkeley National Laboratory are supported by the Director, Office of Science, Office of Basic Energy Sciences, and U.S. Department of Energy under Contract No. DE-AC02-05CH11231.

Q.L. and M.Y. contributed equally to this work. 
[1] J. Nogues and I. K. Schuller, J. Magn. Magn. Mater. 192, 203 (1999).

[2] M. Kiwi, J. Magn. Magn. Mater. 234, 584 (2001).

[3] W. Zhang and K. M. Krishnan, Mater. Sci. Eng. R Rep. 105, 1 (2016).

[4] A. K. Nayak, M. Nicklas, S. Chadov, P. Khuntia, C. Shekhar, A. Kalache, M. Baenitz, Y. Skourski, V. K. Guduru, A. Puri, U. Zeitler, J. M. D. Coey, and C. Felser, Nat. Mater. 14, 679 (2015).

[5] K. Yang, W. Paul, F. D. Natterer, J. L. Lado, Y. Bae, P. Willke, T. Choi, A. Ferrón, J. Fernández-Rossier, A. J. Heinrich, and C. P. Lutz, Phys. Rev. Lett. 122, 227203 (2019).

[6] F. Y. Yang and C. L. Chien, Phys. Rev. Lett. 85, 2597 (2000).

[7] S. Brems, K. Temst, and C. Van Haesendonck, Phys. Rev. Lett. 99, 067201 (2007).

[8] H. Ouyang, K.-W. Lin, C.-C. Liu, S.-C. Lo, Y.-M. Tzeng, J.-Y. Guo, and J. van Lierop, Phys. Rev. Lett. 98, 097204 (2007).

[9] X. P. Qiu, D. Z. Yang, S. M. Zhou, R. Chantrell, K. O'Grady, U. Nowak, J. Du, X. J. Bai, and L. Sun, Phys. Rev. Lett. 101, 147207 (2008).

[10] J. Sort, A. Hoffmann, S.-H. Chung, K. S. Buchanan, M. Grimsditch, M. D. Baró, B. Dieny, and J. Nogués, Phys. Rev. Lett. 95, 067201 (2005).

[11] A. Scholl, M. Liberati, E. Arenholz, H. Ohldag, and J. Stohr, Phys. Rev. Lett. 92, 247201 (2004).

[12] W. H. Meiklejohn, J. Appl. Phys. 33, 1328 (1962).

[13] R. Morales, Z.-P. Li, J. Olamit, K. Liu, J. M. Alameda, and I. K. Schuller, Phys. Rev. Lett. 102, 097201 (2009).

[14] M. Y. Khan, C.-B. Wu, and W. Kuch, Phys. Rev. B 89, 094427 (2014).

[15] Y. Xu, Q. Ma, J. W. Cai, and L. Sun, Phys. Rev. B 84, 054453 (2011).

[16] A. Migliorini, B. Kuerbanjiang, T. Huminiuc, D. Kepaptsoglou, M. Muñoz, J. L. F. Cuñado, J. Camarero, C. Aroca, G. VallejoFernández, V. K. Lazarov, and J. L. Prieto, Nat. Mater. 17, 28, (2018).

[17] H. Ohldag, A. Scholl, F. Nolting, E. Arenholz, S. Maat, A. T. Young, M. Carey, and J. Stöhr, Phys. Rev. Lett. 91, 017203 (2003).

[18] S. Bruck, G. Schütz, E. Goering, X. Ji, and K. M. Krishnan, Phys. Rev. Lett. 101, 126402 (2008).

[19] J. Wu, J. S. Park, W. Kim, E. Arenholz, M. Liberati, A. Scholl, Y. Z. Wu, C. Hwang, and Z. Q. Qiu, Phys. Rev. Lett. 104, 217204 (2010).
[20] J. Li, Y. Meng, J. S. Park, C. A. Jenkins, E. Arenholz, A. Scholl, A. Tan, H. Son, H. W. Zhao, C. Hwang, Y. Z. Wu, and Z. Q. Qiu, Phys. Rev. B 84, 094447 (2011).

[21] Q. Li, G. Chen, T. P. Ma, J. Zhu, A. T. N'Diaye, L. Sun, T. Gu, Y. Huo, J. H. Liang, R. W. Li, C. Won, H. F. Ding, Z. Q. Qiu, and Y. Z. Wu, Phys. Rev. B 91, 134428 (2015).

[22] D. Mauri, H. C. Siegmann, P. S. Bagus, and E. Kay, J. Appl. Phys. 62, 3047 (1987).

[23] R. L. Stamps, J. Phys. D: Appl. Phys. 33, R247 (2000).

[24] J.-V. Kim, R. L. Stamps, B. V. McGrath, and R. E. Camley, Phys. Rev. B 61, 8888 (2000).

[25] T. C. Schulthess and W. H. Butler, Phys. Rev. Lett. 81, 4516 (1998).

[26] J. Li, A. Tan, S. Ma, R. F. Yang, E. Arenholz, C. Hwang, and Z. Q. Qiu, Phys. Rev. Lett. 113, 147207 (2014).

[27] Y. Z. Wu, Y. Zhao, E. Arenholz, A. T. Young, B. Sinkovic, C. Won, and Z. Q. Qiu, Phys. Rev. B 78, 064413 (2008).

[28] Q. Li, J. H. Liang, Y. M. Luo, Z. Ding, T. Gu, Z. Hu, C. Y. Hua, H.-J. Lin, T. W. Pi, S. P. Kang, C. Won, and Y. Z. Wu, Sci. Rep. 6, 22355 (2016).

[29] J. Li, E. Jin, H. Son, A. Tan, W. N. Cao, C. Hwang, and Z. Q. Qiu, Rev. Sci. Instrum. 83, 033906 (2012).

[30] Q. Li, T. Gu, J. Zhu, Z. Ding, J. X. Li, J. H. Liang, Y. M. Luo, Z. Hu, C. Y. Hua, H.-J. Lin, T. W. Pi, C. Won, and Y. Z. Wu, Phys. Rev. B 91, 104424 (2015).

[31] J. A. De Toro, D. P. Marques, P. Muñiz, V. Skumryev, J. Sort, D. Givord, and J. Nogués, Phys. Rev. Lett. 115, 057201 (2015).

[32] J. A. Borchers, M. J. Carey, R. W. Erwin, C. F. Majkrzak, and A. E. Berkowitz, Phys. Rev. Lett. 70, 1878 (1993).

[33] E. N. Abarra, K. Takano, F. Hellman, and A. E. Berkowitz, Phys. Rev. Lett. 77, 3451 (1996)

[34] J. Wu, D. Carlton, J. S. Park, Y. Meng, E. Arenholz, A. Doran, A. T. Young, A. Scholl, C. Hwang, H. W. Zhao, J. Bokor, and Z. Q. Qiu, Nat. Phys. 7, 303 (2011).

[35] M. Yang, Q. Li, A. T. N'Diaye, Q. Y. Dong, N. Gao, E. Arenholz, C. Hwang, Y. Z. Wu, and Z. Q. Qiu, J. Magn. Magn. Mater. 460, 6 (2018).

[36] J. Li, M. Przybylski, F. Yildiz, X. L. Fu, and Y. Z. Wu, Phys. Rev. B 83, 094436 (2011).

[37] M. Fraune, U. Rüdiger, G. Güntherodt, S. Cardoso, and P. Freitas, Appl. Phys. Lett. 77, 3815 (2000).

[38] W. N. Cao, J. Li, G. Chen, J. Zhu, C. R. Hu, and Y. Z. Wu, Appl. Phys. Lett. 98, 262506 (2011).

[39] See Supplemental Material at http://link.aps.org/supplemental/ 10.1103/PhysRevMaterials.3.114415 the details of chirality switching and energy folding. 